\title{
TAMBOR ROTATÓRIO OPERANDO NO REGIME DE ROLAMENTO: UM ESTUDO EXPERIMENTAL E NUMÉRICO
}

\author{
R. SCATENA ${ }^{1}$, L.C. FERREIRA ${ }^{1}$, D.A. SANTOS $^{1}$, C.R. DUARTE ${ }^{1}$, M.A.S. BARROZO ${ }^{1}$ \\ ${ }^{1}$ Universidade Federal de Uberlândia, Faculdade de Engenharia Química \\ E-mail para contato: rafascatena@gmail.com
}

\begin{abstract}
RESUMO - No presente trabalho foram realizados, tanto um estudo experimental quanto um estudo numérico, da dinâmica de partículas no interior de um tambor rotatório operando no regime de rolamento. A abordagem multifásica Euler-Euler, juntamente com a teoria cinética do escoamento granular, foi utilizada em todas as simulações. No intuito de se obter, experimentalmente, os perfis de velocidade de partículas, foi proposto a utilização de uma técnica não intrusiva composta por uma câmera de alta velocidade. Os resultados das simulações mostraram-se satisfatórios quando comparados aos dados experimentais e possibilitaram a análise da influência da velocidade de rotação e da fração de preenchimento do tambor rotatório sobre a velocidade das partículas e espessura da camada ativa presentes no escoamento.
\end{abstract}

\section{INTRODUÇÃO}

Muitos setores industriais tais como, mineração, metalurgia, processamento de alimentos, polímeros e indústrias bioquímicas, possuem processos que requerem uma elevada área de contato entre as fases envolvidas, no intuito de aumentar as taxas de transferência de massa e energia. Desta forma, os tambores rotatórios surgem como equipamentos adequados no processamento de materiais granulares em aplicações tais como, mistura, granulação, moagem, recobrimento e secagem (Degrève et al., 2006; Dubé et al., 2013).

Tambores rotatórios são largamente empregados nos setores industriais, principalmente, devido a sua capacidade de lidar com materiais contendo uma larga faixa de distribuição granulométrica e diferenças significativas nas suas propriedades físicas.

Como observado por Mellmann (2001), dependendo da velocidade de rotação do tambor, de sua geometria, da fração de preenchimento e das propriedades físicas do material particulado, tambores rotatórios podem apresentar, basicamente, cinco diferentes regimes de escoamento granular em seu interior (escorregamento, rolamento, cascateamento, catarateamento e centrifugação), cada qual contendo um comportamento dinâmico intrínseco, o que aumenta ainda mais a complexidade em seu estudo.

Logo, muitos trabalhos têm sido desenvolvidos em tambores rotatórios no intuito de se estudar: 
os diferentes regimes de escoamentos granulares (Mellmann, 2001; Watanabe, 1999), o fenômeno de segregação e mistura de partículas (Lee et al., 2013; Ding et al., 2002) e o comportamento dinâmico de partículas (Dubé et al., 2013; Ding et al., 2001; Boateng e Barr, 1997).

Dentre todos os regimes, o regime de rolamento é o mais comumente utilizado na indústria. Como pode ser observado na Figura 1, este regime é caracterizado por possuir duas diferentes regiões: uma região passiva, próxima à parede do tambor, onde as partículas se movem como um corpo sólido, e uma região ativa, próxima à superfície do leito de material, onde ocorrem os principais mecanismos de mistura e transferência de massa e energia (Dubé et al., 2013; Ding et al., 2001).



Figura 1 - Esquema das regiões ativa e passiva presentes no regime de rolamento em um tambor rotatório.

O escoamento granular se inverte quando da passagem da região passiva para a região ativa, o que caracteriza a interface ativo-passiva. A distância entre a interface ativo-passiva e a superfície do leito de partículas é definida como sendo a espessura da camada ativa. Existem diferentes técnicas, denominadas intrusivas e não intrusivas, para a medida da velocidade de partículas.

Técnicas não intrusivas, que utilizam detectores de raios- $\gamma$ para a quantificação das velocidades das partículas através do monitoramento de suas posições, foram utilizadas, dentre outros pesquisadores, por Ding et al. (2002) e Dubé et al. (2013). Entretanto, tais métodos são aplicáveis apenas a partículas radioativas.

Nakagawa et al. (1993) e Yada et al. (2010) utilizaram imagens de ressonância magnética como técnica não intrusiva para o estudo do movimento de partículas no interior de um tambor rotatório.

Alguns trabalhos na literatura vêm demostrando a viabilidade em se estudar escoamentos 


\section{9 a 22 de outubro de 2014 \\ Florianópolis/SC}

granulares por meio de uma técnica não intrusiva composta por uma câmera de alta velocidade (Duarte et al., 2005). Após a gravação do escoamento granular, a velocidade da partícula é calculada baseando-se na distância percorrida e no respectivo número de frames.

Por outro lado, Boateng e Barr (1997) utilizaram, como técnica intrusiva, sondas de fibra ótica para medir as principais propriedades do escoamento em um tambor rotatório. Contudo, tais sondas podem causar, dependendo de suas geometrias, perturbações no escoamento e, consequentemente, erros de medição.

Paralelamente aos estudos experimentais, as simulações numéricas surgem como uma importante ferramenta complementar no estudo de escoamentos granulares. A abordagem Euler-Euler e o Método dos Elementos Discretos (DEM) são os principais métodos utilizados nas simulações de escoamentos granulares.

Na metodologia DEM, cada partícula presente no escoamento é acompanhada e todas as forças de interação que agem sobre elas são calculadas individualmente. Embora muitos autores tenham adotado este tipo de metodologia (Marigo et al., 2012; Kwapinska et al., 2006), a mesma ainda se encontra restrita ao número de partículas presentes no interior do equipamento devido ao alto custo computacional.

Por outro lado, na abordagem Euler-Euler, ambas as fases são tratadas como contínuas e interpenetrantes e resolvidas em um campo Euleriano. Simulações numéricas utilizando a abordagem Euler-Euler vêm se tornando cada vez mais comum no estudo de escoamentos granulares em diferentes tipos de equipamentos (Santos et al., 2012a; Santos et al., 2012b; Cunha et al., 2009; Barrozo et al., 2010; Santos et al., 2009; Oliveira et al., 2009; Duarte et al., 2009).

Desta forma, o presente trabalho tem por objetivo estudar a dinâmica de partículas em um tambor rotatório, operando no regime de rolamento e submetido a diferentes condições operacionais, por meio de experimentos e simulações numéricas.

\section{MATERIAIS E MÉTODOS}

\subsection{Metodologia Experimental}

O tambor rotatório utilizado no presente trabalho foi construído em aço inox contendo $50 \mathrm{~cm}$ de comprimento e $21,5 \mathrm{~cm}$ de diâmetro interno. A parede frontal foi confeccionada em vidro transparente para possibilitar a visualização do escoamento granular em seu interior. No intuito de se evitar o deslizamento do material sobre a parede do tambor, o interior do mesmo foi revestido com uma lixa (P80).

Foram utilizados, como material particulado, grãos de soja com diâmetro de 6,2 mm e densidade de $1164 \mathrm{~kg} / \mathrm{m}^{3}$. O limite máximo de empacotamento (concentração de sólidos inicial) para os grãos de soja foi de $60 \%$ (porcentagem em volume).

Os perfis de velocidade de partículas, na metade da corda do leito de material (linha de 
referência na Figura 1), foram medidos por meio de uma câmera de alta velocidade (até 2000 frames/s). As posições das partículas foram determinadas através do software analisador de imagens Meazure $^{\mathrm{TM}}$ e o tempo necessário para transpor esta distância foi calculado como base nos respectivos frames.

Foram investigadas, no presente trabalho, as seguintes condições operacionais: frações de preenchimento de $18,81 \%$ e $31,40 \%$ e velocidades de rotação do tambor de $0,712 \mathrm{rad} / \mathrm{s}$ e $1,45 \mathrm{rad} / \mathrm{s}$.

\subsection{Metodologia Numérica}

A abordagem multifásica Euler-Euler, juntamente com a teoria cinética do escoamento granular, utilizada no presente trabalho, é descrita com detalhes por Duarte et al. (2005).

As seguintes condições foram adotadas nas simulações computacionais: malha computacional contendo 300.000 células; simulações tridimensionais (3D); condição de contorno de não deslizamento (no-slip) na parede do tambor; algoritmo de acoplamento pressão-velocidade SIMPLE; esquema de interpolação espacial $U p$-wind de primeira ordem; tempo total de simulação de $60 \mathrm{~s}$ para cada condição; passo no tempo (time step) de $1 \times 10^{-3}$; critério de convergência de $1 \times 10^{-3}$.

\section{RESULTADOS E DISCUSSÕES}

No intuito de se investigar a dinâmica de partículas em um tambor rotatório operando no regime de rolamento, perfis de velocidades de partículas na metade da corda do leito de material (linha de referência na Figura 1) foram medidos, experimentalmente, por meio de uma câmera de alta velocidade e comparados, sob as mesmas condições, com as simulações numéricas. As Figuras 2 e 3 mostram os resultados dos perfis de velocidade para uma fração de preenchimento de $18,81 \%$ e velocidades de rotação de 0,712 e $1,45 \mathrm{rad} / \mathrm{s}$, respectivamente.
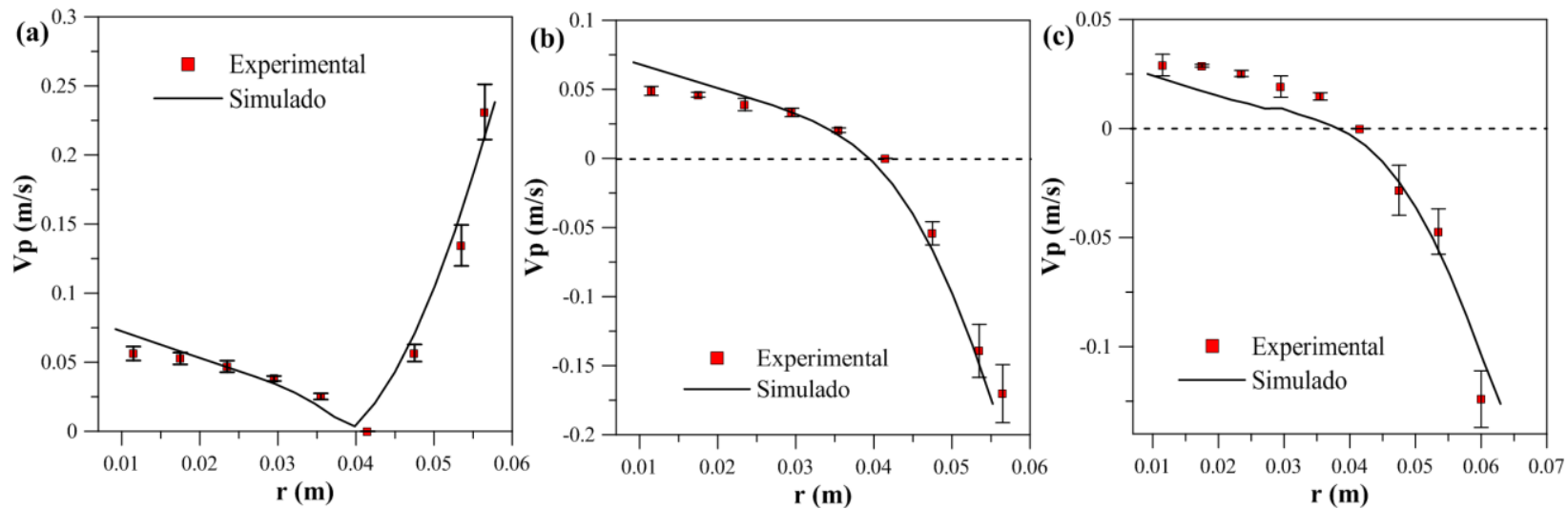

Figura 2 - Perfis de velocidade de partículas: fração de preenchimento de 18,81\% e velocidade de rotação de $0,712 \mathrm{rad} / \mathrm{s}$ - (a) velocidade resultante; (b) velocidade na direção x; (c) velocidade na direção y. 

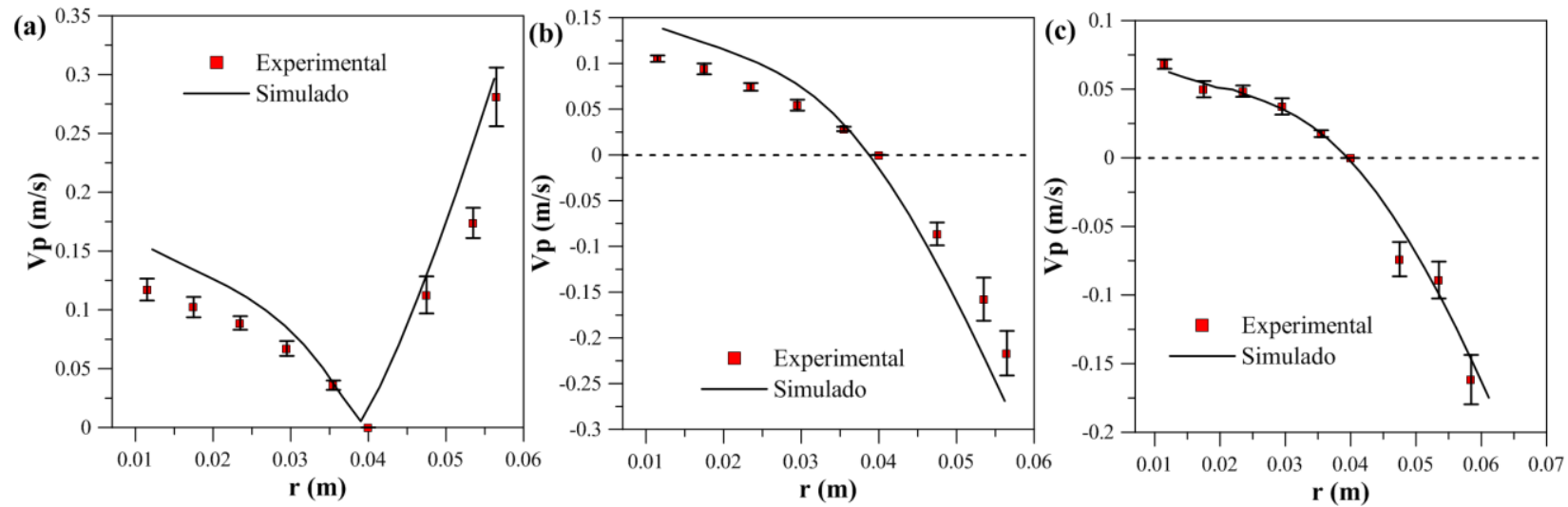

Figura 3 - Perfis de velocidade de partículas: fração de preenchimento de $18,81 \%$ e velocidade de rotação de 1,45 rad/s - (a) velocidade resultante; (b) velocidade na direção x; (c) velocidade na direção y.

Pode-se observar (Figuras 2 e 3) uma satisfatória correspondência entre os dados experimentais e os respectivos resultados numéricos. Em todas as condições nota-se que, o maior valor de velocidade de partícula se encontra próximo à superfície do leito de material (região ativa), decresce com a diminuição da posição radial e alcança um valor nulo na interface ativo-passiva, onde o escoamento granular é invertido devido ao movimento da parede do tambor.

As figuras 4 e 5 mostram comportamentos semelhantes como os elucidados anteriormente, porém, para uma fração de preenchimento de $31,40 \%$ e velocidades de rotação de 0,712 e $1,45 \mathrm{rad} / \mathrm{s}$, respectivamente.
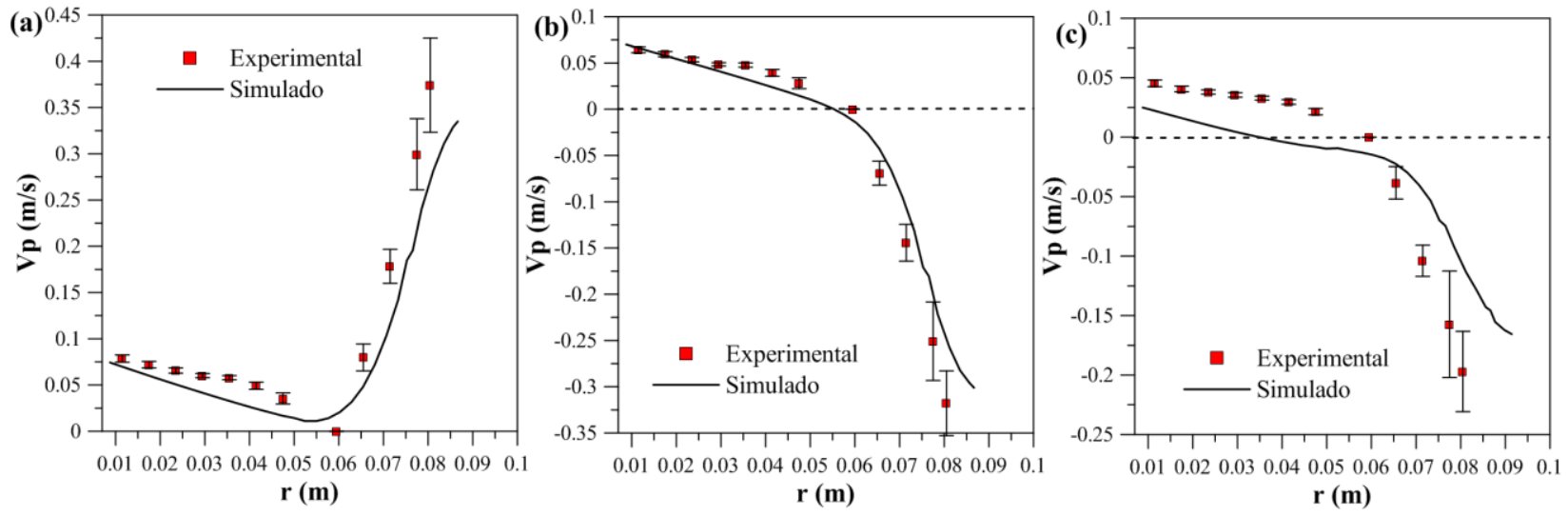

Figura 4 - Perfis de velocidade de partículas: fração de preenchimento de 31,40\% e velocidade de rotação de $0,712 \mathrm{rad} / \mathrm{s}$ - (a) velocidade resultante; (b) velocidade na direção x; (c) velocidade na direção y. 


\section{9 a 22 de outubro de 2014 \\ Florianópolis/SC}
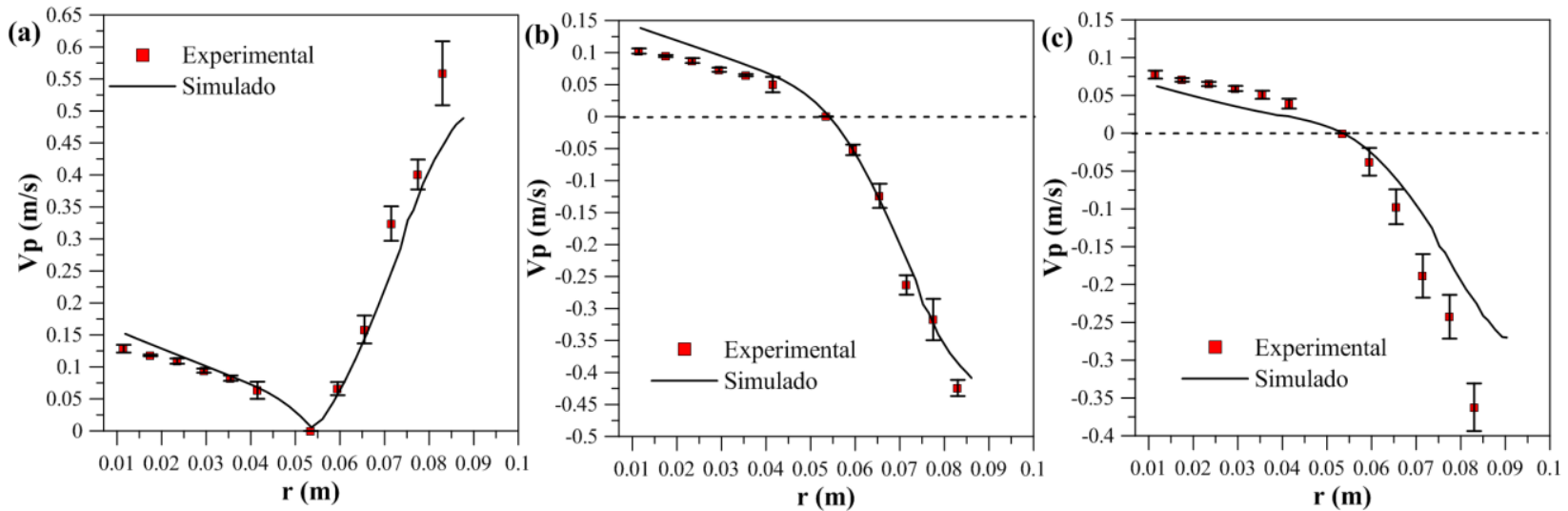

Figura 5 - Perfis de velocidade de partículas: fração de preenchimento de 31,40\% e velocidade de rotação de 1,45 rad/s - (a) velocidade resultante; (b) velocidade na direção x; (c) velocidade na direção y.

Como esperado, duas diferentes regiões presentes no escoamento transversal em um tambor rotatório foram visualizadas: uma região ativa, próxima à superfície livre, se movimentando com uma velocidade relativamente alta e uma região passiva, próxima à parede do tambor, a qual se movimenta proporcionalmente à velocidade de rotação do tambor.

Nota-se que, fixando-se o valor da fração de preenchimento e aumentando-se o valor da velocidade de rotação do tambor, ocorreu um aumento na magnitude da velocidade das partículas tanto na região ativa quanto na região passiva. Contudo, a posição do ponto de inversão do sentido da velocidade (interface ativo-passiva) permaneceu constante (Figuras 2-5).

Como pode ser observado, o ponto de inversão da velocidade ocorreu a aproximadamente 4,0 $\mathrm{cm}$ da parede do tambor, para a fração de preenchimento de $18,81 \%$, independentemente da velocidade de rotação do tambor (Figuras 2 e 3). Já para a fração de preenchimento de 31,40\%, o ponto de inversão ocorreu a $5,5 \mathrm{~cm}$ da parede do tambor, independentemente da velocidade de rotação (Figuras 4 e 5).

Por outro lado, fixando-se a velocidade de rotação do tambor e aumentando-se a fração de preenchimento, ocorreu um aumento na magnitude da velocidade das partículas apenas na região ativa. Pode ser notado, também que, a posição do ponto de inversão moveu-se de 4,0 para $5,5 \mathrm{~cm}$ da parede do tambor, ou seja, ocorreu um aumento na espessura da região passiva (Figuras 2-5). Quanto maior a velocidade das partículas na região ativa, maior é a possibilidade de transição para outros regimes de escoamento. Logo, como observado por Mellmann (2001), dentre outras variáveis, um aumento na velocidade de rotação ou na fração de preenchimento pode conduzir à transição para os demais regimes em um tambor rotatório.

A espessura da camada ativa, medida experimentalmente, para a fração de preenchimento de $18,81 \%$ e velocidades de rotação de $0,712 \mathrm{rad} / \mathrm{s}$ e $1,45 \mathrm{rad} / \mathrm{s}$, foram de $1,5 \mathrm{~cm}$ e $1,65 \mathrm{~cm}$, respectivamente (Figuras 2 e 3). Para a fração de preenchimento de 31,40\%, os valores foram de 2,10 $\mathrm{cm}$ e $2,95 \mathrm{~cm}$, respectivamente para velocidades de rotação de $0,712 \mathrm{rad} / \mathrm{s}$ e 1,45 rad/s (Figuras 4 e 5 ). 


\section{9 a 22 de outubro de 2014 \\ Florianópolis/SC}

Desta forma, um aumento na velocidade de rotação causou um aumento na espessura da região ativa. Este efeito foi mais acentuado quando da utilização da maior fração de preenchimento $(31,40 \%)$. Logo, quanto maior for a velocidade de rotação ou a fração de preenchimento, maior a espessura da região ativa e, consequentemente, maiores as taxas de transferência de massa, energia e quantidade de movimento.

Os perfis de velocidade na região ativa não foram parabólicos, mas, ao contrário, foram essencialmente lineares. Este resultado está de acordo com aqueles observados por Boateng e Barr (1997), os quais sugeriram que, na maior parte da região ativa a tensão de cisalhamento é uniforme $(d u / d y=$ constante $)$.

\section{CONCLUSÕES}

Foi possível, através de um estudo experimental e numérico, investigar o comportamento dinâmico das partículas no interior de um tambor rotatório operado no regime de rolamento. As simulações numéricas utilizando a abordagem Euler-Euler se mostraram satisfatórias quando comparadas com os dados experimentais sob as mesmas condições.

Observou-se uma grande influência da velocidade de rotação e da fração de preenchimento do tambor sobre a espessura da camada ativa, a qual está diretamente relacionada às taxas de transferência de massa e energia.

\section{AGRADECIMENTOS}

Agradecemos a Fundação de Amparo à Pesquisa do Estado de Minas Gerais (FAPEMIG) pelos recursos concedidos no Projeto de Participação Coletiva em Eventos Técnico-Científicos (PCE00082-14). Os autores agradecem, também, à CAPES e ao CNPq pelo apoio financeiro concedido por meio de bolsas de pesquisa.

\section{REFERÊNCIAS}

BARROZO, M.A. S.; DUARTE, C.R.; EPSTEIN, N.; GRACE, J. R.; LIM, C.J. Experimental and CFD study of dense-phase, transition region and dilute-phase spouting. Ind. Eng. Chem. Res., v.49, p.5102-5109, 2010.

BOATENG, A.A.; BARR, P.V. Granular flow behaviour in the transverse plane of a partially filled rotating cylinder. J. Fluid Mech., v.330, p.233-249, 1997.

CUNHA, F.G.; SANTOS, K.G.; ATAÍDE, C.H.; EPSTEIN, N.; BARROZO, M.A.S. Annatto powder production in a spouted bed: An experimental and CFD study. Ind. Eng. Chem. Res., v.48, p.976-982, 2009.

DEGRÈVE, J.; BAEYENS, J.; VAN DE VELDEN, M.; DE LAET, S. Spray-agglomeration of NPKfertilizer in a rotating drum granulator. Powder Technol., v.163, p.188-195, 2006.

DING, Y.L.; FORSTER, R.; SEVILLE, J.P.K.; PARKER, D.J. Segregation of granular flow in the transverse plane of a rolling mode rotating drum. Int. J. Multiphase Flow, v.28, p.635-663, 2002. 

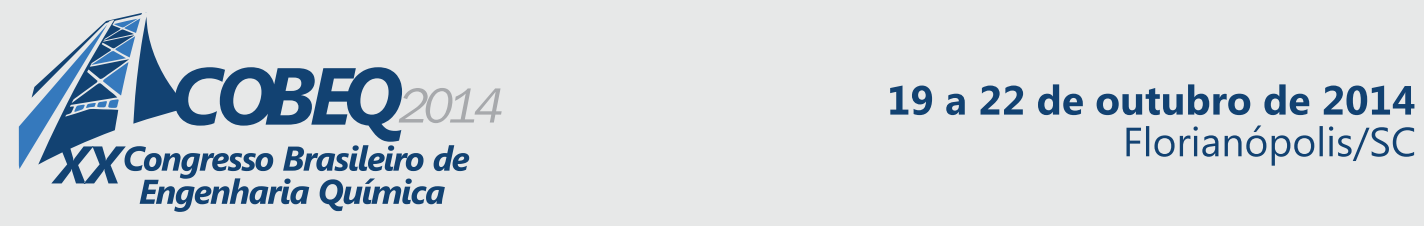

DING, Y.L.; SEVILLE, J.P.K.; FORSTER, R.; PARKER, D.J. Solids motion in rolling mode rotating drums operated at low to medium rotational speeds. Chem. Eng. Sci., v.56, p.1769-1780, 2001.

DUARTE, C.R.; MURATA, V.V.; BARROZO, M. A. S. A study of the fluid dynamics of the spouted bed using CFD. Braz. J. Chem. Eng., v.22, p.263-270, 2005.

DUARTE, C.R.; OLAZAR, M.; MURATA, V.V.; BARROZO, M.A.S. Numerical simulation and experimental study of fluid-particle flows in a spouted bed. Powder Technol., v.188, p.195-205, 2009.

DUBÉ, O.; ALIZADEH, E.; CHAOUKI, J.; BERTRAND, F. Dynamics of non-spherical particles in a rotating drum. Chem. Eng. Sci., v.101, p.486-502, 2013.

KWAPINSKA, M.; SAAGE, G.; TSOTSAS, E. Mixing of particles in rotary drums: A comparison of discrete element simulations with experimental results and penetration models for thermal processes. Powder Technol., v.161, p.69-78, 2006.

LEE, C.-F.; CHOU, H.-T.; CAPART, H. Granular segregation in narrow rotational drums with different wall roughness: Symmetrical and asymmetrical patterns. Powder Technol., v.233, p.103$115,2013$.

MARIGO, M.; CAIRNS, D.L.; DAVIES, M.; INGRAM, A.; STITT, E.H. A numerical comparison of mixing efficiencies of solids in a cylindrical vessel subject to a range of motions. Powder Technol., v.217, p.540-547, 2012.

MELLMANN, J. The transverse motion of solids in rotating cylinders-forms of motion and transition behavior. Powder Technol., v.118, p.251-270, 2001.

NAKAGAWA, M.; ALTOBELLI, S.A.; CAPRIHAN, A.; FUKUSHIMA, E.; JEONG, E.-K. Noninvasive measurements of granular flows by magnetic resonance imaging. Exp. Fluids, v.16, p.54-60, 1993.

OLIVEIRA, D.C.; ALMEIDA, C.A.K.; VIEIRA, L.G.M.; DAMASCENO, J.J.R.; BARROZO, M.A.S. Influence of geometric dimensions on the performance of a filtering hydrocyclone: an experimental and CFD study. Braz. J. Chem. Eng., v.26, p.575-582, 2009.

SANTOS, D.A.; ALVES, G.C.; DUARTE, C.R.; BARROZO, M.A.S. Disturbances in the hydrodynamic behavior of a spouted bed caused by an optical fiber probe: Experimental and CFD study. Ind. Eng. Chem. Res., v.51, p.3801-3810, 2012a.

SANTOS, K.G.; MURATA, V.V.; BARROZO, M.A.S. Three-dimensional computational fluid dynamics modeling of spouted bed. Can. J. Chem. Eng., v.87, p.211-219, 2009.

SANTOS, K.G.; SANTOS, D.A.; DUARTE, C.R.; MURATA, V.V.; BARROZO, M.A.S. Spouting of bidisperse mixture of particles: A CFD and experimental study. Drying Technol., v.30, p.1354$1367,2012 b$.

WATANABE, H. Critical rotation speed for ball-milling. Powder Technol.. v.104, p.95-99, 1999.

YADA, H.; KAWAGUCHI, T.; TANAKA, T. Relation between segregation patterns and granular flow modes in conical rotating drum. Flow Meas. Instrum., v.21, p.207-211, 2010. 